\title{
Tensiones constitutivas entre el diseño y la implementación del currículo en la enseñanza de las Ciencias Naturales en el contexto escolar colombiano
}

\author{
Villanueva Meneses, Rodolfo; González Melo, Hamlet Santiago \\ Tensiones constitutivas entre el diseño y la implementación del currículo en la enseñanza de las Ciencias \\ Naturales en el contexto escolar colombiano \\ Revista Educación, vol. 45, núm. 1, 2021 \\ Universidad de Costa Rica, Costa Rica \\ Disponible en: http://www.redalyc.org/articulo.oa?id=44064134003 \\ DOI: https://doi.org/10.15517/revedu.v45i1.40620
}

Esta obra está bajo una Licencia Creative Commons Atribución-NoComercial-SinDerivar 3.0 Internacional. 


\title{
Tensiones constitutivas entre el diseño y la implementación del currículo en la enseñanza de las Ciencias Naturales en el contexto escolar colombiano
}

\begin{abstract}
Constitutive Tensions in Curriculum Design and Implementation for Science Teaching within the Context of a Colombian School
\end{abstract}

Rodolfo Villanueva Meneses

Universidad de La Sabana, Colombia

rvillanueva@educacionbogota.edu.co

http://orcid.org/0000-0002-1398-2993

Hamlet Santiago González Melo

Universidad Distrital Francisco José de Caldas, Colombia

hsgonzalezm@udistrital.edu.co

iD http://orcid.org/0000-0003-1210-8679
DOI: https://doi.org/10.15517/revedu.v45i1.40620

Redalyc: http://www.redalyc.org/articulo.oa?id=44064134003

Recepción: 13 Marzo 2020

Aprobación: 13 Agosto 2020

\section{RESUMEN:}

La transición entre las políticas educativas y su aplicación real en las aulas constituye un tema que promueve diferentes e interesantes reflexiones en el campo de la enseñanza de las Ciencias Naturales en Colombia. La discusión sobre los niveles de concreción curricular debe ir más allá de la mera crítica, para convertirse en un factor de reflexión el cual sitúe a la persona educadora en el campo de la producción académica a través del análisis de la teoría y de la reflexión de su práctica pedagógica. En ese marco, el presente artículo pretende escudriñar a nivel teórico algunas concepciones del currículo de Ciencias, su articulación con las políticas nacionales y, problemáticas derivadas de su puesta en funcionamiento. Es así como al inicio se expone una breve reseña histórica del currículo de Ciencias en Colombia, para luego dar lugar a los lineamientos curriculares que operan en la actualidad y desarrollar algunas reflexiones en torno a las implicaciones de estos tránsitos y las tensiones con su puesta en marcha por parte del cuerpo docente. Para finalizar, se analizan algunas situaciones específicas en donde las tensiones entre lo planeado y ejecutado dan pie al planteamiento de una reflexión propositiva en términos de la resolución de las dificultades en la cotidianidad del profesorado.

Palabras Clave: Enseñanza, Aprendizaje, Currículo, Ciencias Naturales.

\section{Abstract:}

Practical application of educational policies in the classroom tends to unleash a gamut of diverse reactions and insights, particularly with regards to how teaching Colombia's Natural Science curriculum is carried out. Successful implementation of the any curriculum requires transcending beyond simple criticism in order to stir thought in the student based on theoretical analysis and review by the teacher of their pedagogical experience. This study examines, on a theoretical level, certain notions associated with the Natural Sciences curriculum, its articulation with Colombian educational policies and implementation issues that arise. The article briefly summarizes the historical background of the curriculum to the present-day constraints among the teachers that must implement it. Analysis of different scenarios that examine curriculum planning and execution attempt to propose alternatives to help teachers cope with the aforementioned issues in their daily practice.

KEYWORDS: Teaching, Learning, Curriculum, Natural Sciences.

\section{INTRODUCCIÓN}

$\mathrm{Al}$ revisar los aspectos que permean los procesos curriculares en la enseñanza de las Ciencias Naturales y que influyen en su implementación, se consideran tanto los de origen externo como interno de las instituciones educativas, tales como: las transformaciones del currículo de Ciencias en Colombia desde el marco legal de referencia; las condiciones operativas, organizacionales y administrativas de las instituciones; la influencia de 
la familia y la sociedad durante el desarrollo del proceso; la forma como el cuerpo docente concibe, diseña, implementa y evalúa el currículo; y la forma en que el estudiantado influye en todo el proceso educativo. En tal sentido, se hace especialmente referencia a los factores asociados al profesorado y las instituciones educativas visto desde el panorama general del currículo de Ciencias Naturales en Colombia, las transformaciones en el diseño curricular de Ciencias y las transformaciones producto de su implementación.

Casanova (2006) indica que los modelos curriculares pueden ser abiertos o cerrados, los primeros posibilitan la flexibilidad de los procesos y corresponden a las sociedades democráticas, pluralistas, participativas, diversas e interculturales; estas características del currículo se complementan con las señaladas por Rivera (2010) cuando expresa que el currículo legal debe ser abierto, inclusivo, flexible, pensado desde la diversidad y generador de personal docente reflexivo. En cambio, los segundos son rígidos en la proposición de sus elementos. Al respecto, Glatthorn, Boschee, Boschee y Whitehead (2018) mencionan varios tipos de currículo: recomendado, escrito, apoyado, enseñado, evaluado y aprendido. Además, reconocen su intencionalidad, diferenciado del currículo oculto pues no es producto de una intención. Rivera (2010) sostiene que el currículo se desarrolla en un proceso cíclico o dinámico que comprende seis etapas: planeación, diseño, revisión, ejecución, verificación y validación. Estas etapas sirven como referente para hacer seguimiento al desarrollo curricular y las situaciones que dificultan su transición.

Las transformaciones curriculares son un fenómeno recurrente a través del tiempo, se presentan con frecuencia a partir de los conceptos del profesorado y directivos/as docentes. A nivel de la enseñanza de las ciencias, en efecto, existen notables diferencias entre lo establecido, inicialmente por el Estado, en materia de las orientaciones curriculares y, lo que realmente aprende el alumnado sobre Ciencias.

\section{El currículo de Ciencias Naturales en Colombia}

En Colombia, el currículo de Ciencias Naturales ha sido una construcción que se evidencia desde la revisión de los cambios establecidos en el marco legal por el Estado y, las orientaciones curriculares planteadas a nivel nacional y regional. Por tal razón, es importante tener un panorama general de estas transformaciones.

\subsection{Referentes curriculares en Ciencias Naturales}

Las orientaciones curriculares en Ciencias Naturales y educación ambiental para Colombia se encuentran en los lineamientos curriculares, los Estándares Básicos en Competencias y, la nueva propuesta curricular sobre los Derechos Básicos de los Aprendizajes y las mallas de aprendizaje.

En los lineamientos curriculares, definidos por el Ministerio de Educación Nacional de Colombia (MEN) en 1998, se fijaron los referentes filosóficos, epistemológicos, sociológicos y psicocognitivos; sus implicaciones pedagógicas y didácticas y, la propuesta de contenidos básicos para cada grado escolar (MEN, 1998). No obstante, los lineamientos tienen más de dos décadas, por lo tanto debieron ser evaluados y actualizados conforme el conocimiento científico y tecnológico se deconstruye y nuevo conocimiento surge con el tiempo.

En el año 2004, el Ministerio de Educación Nacional publicó los Estándares Básicos en Competencias (EBC), los cuales contienen una transformación de las propuestas curriculares del pasado enfocadas en los contenidos, intentando ampliar esta visión hacia el desarrollo de competencias; ya no se trata solamente del saber, también, se reconoce la importancia del saber hacer y desarrollar en estudiantes las habilidades científicas: explorar hechos y fenómenos; analizar problemas; observar, recoger y analizar información relevante; utilizar diferentes métodos de análisis; evaluar los métodos, y compartir los resultados. Según el Ministerio "en un entorno cada vez más complejo, competitivo y cambiante, formar en Ciencias significa contribuir a la formación de ciudadanos y ciudadanas capaces de razonar, debatir, producir, convivir y desarrollar al máximo su potencial creativo” (MEN, 2004, p. 6). La estructura curricular de los EBC se 
fundamenta en tres ejes de procesos: Me aproximo al conocimiento como científico natural, manejo de conocimientos propios de las Ciencias Naturales y, desarrollo compromisos personales y sociales. El eje sobre conocimientos propios se subdivide en entorno vivo, entorno físico, y ciencia, tecnología y sociedad; en décimo y undécimo el componente de entorno físico se discrimina en procesos químicos y físicos. En cada uno de los ejes se muestran las acciones de pensamiento y de producción concretas que el estudiantado debe desarrollar desde primero hasta undécimo; no obstante, se presentan por conjuntos de grados, así: primero a tercero, cuarto a quinto, sexto a séptimo, octavo a noveno y, décimo a undécimo (MEN, 2004).

$\mathrm{Al}$ revisar los estándares en Ciencias se encuentran aspectos para revaluar. En primer lugar, las competencias planteadas están definidas por grupos de grados y no por separado para cada grado, por lo que, resultan muy generales al momento de implementarlas para algún grado en particular. Esta situación se ve reflejada cuando el alumnado debe ir de una institución educativa a otra, puesto que, muchos establecimientos no coinciden en las competencias que desarrollan o en el nivel alcanzado en cada una de estas; de paso, se podrían generar dificultades tanto para estudiantes como para docentes, pues se debe pensar en la forma de nivelar el desarrollo o fortalecimiento de las competencias que se requieran. En segundo lugar, han surgido nuevos estudios sobre cada una de las competencias científicas y niveles de desarrollo, los cuales, sirven como punto de partida para definir niveles que se pueden plantear para cada grado o determinados grados.

En el año 2016, se publicó la primera versión de los Derechos Básicos de Aprendizaje (DBA) en Ciencias Naturales y Ciencias Sociales, y la segunda versión en Matemáticas y Lenguaje. Estas propuestas curriculares fueron dirigidas a docentes y directivos/as docentes, y se complementaron con las mallas de aprendizaje en estas asignaturas. Según el Ministerio de Educación Nacional de Colombia (2016), los derechos y mallas de aprendizaje en Ciencias se fundamentan en soportes teóricos y conceptuales, la revisión de documentos curriculares nacionales e internacionales, las pruebas estandarizadas y, el marco legal:

Los dos referentes de actualización curricular (DBA y las mallas de aprendizaje) brindan algunas ideas para la promoción de actitudes científicas y ambientales, que buscan ayudar en la comprensión de la naturaleza de la ciencia, los procesos que siguen los científicos, y al desarrollo de valores, actitudes y acciones en correspondencia con premisas de convivencia, democracia, solidaridad y sostenibilidad ambiental. (p. 60)

En Ciencias, las mallas están estructuradas desde los aprendizajes y las habilidades científicas y, constituyen una guía para las consideraciones didácticas y las situaciones de aprendizaje desde la formulación de preguntas para fomentar procesos de pensamiento, las ideas previas y la construcción del conocimiento a partir del diálogo utilizando el lenguaje propio de las ciencias (MEN, 2017). Algunos de los elementos destacables de la propuesta se hallan en el planteamiento de las mallas de aprendizaje para cada grado y su articulación con el grado inmediatamente anterior y superior y; las evidencias de aprendizaje de cada grado que pueden servir para definir los criterios de evaluación. Una dificultad es que las mallas están planteadas hasta quinto grado $\left(5^{\circ}\right)$ limitando su alcance en básica secundaria y media vocacional. De igual manera, los Derechos Básicos de Aprendizaje, en cuanto a la formulación de competencias ofrecen poca información novedosa, pues están muy alineados con los Estándares Básicos por Competencias.

Al contrastar algunos de los planteamientos establecidos en las diferentes orientaciones curriculares para las ciencias en Colombia, se observa una tendencia a proponer el desarrollo de los contenidos y procesos del pensamiento de una manera progresiva, la cual, debe pensarse y analizarse desde nuevos estudios para que se considere mucho mejor la evolución de las dimensiones biológica, lingüística, psicológica y social. Resulta importante destacar, que incluso, algunas de las acciones de pensamiento o procesos para desarrollar habilidades tanto científicas como transversales en el estudiantado, se pueden categorizar y ejecutar en varios grados pues no son exclusivos de un ciclo de formación en particular. Procesos como la indagación, la observación, la argumentación, el desarrollo lectoescritor, entre otros, han sido estudiados por diversos investigadores, por lo que se han diseñado escalas o categorías para definir niveles en cada una de esas acciones 
de pensamiento. Estas escalas varían según la mirada de los investigadores y el campo de conocimiento, y no aplican exactamente igual dentro de campos disciplinares como Ciencias Naturales, Matemáticas u otros.

A continuación, se relacionan algunas competencias en Ciencias Naturales, reconociendo que para cada una de las habilidades de pensamiento y competencias nombradas hay diferentes propuestas. Diversos autores han propuesto matrices sobre los niveles de indagación, por ejemplo, García y Furman (2014) definen unas categorías de análisis para los tipos de preguntas formuladas por un grupo de estudiantes de sexto grado, para ello, hacen una adaptación de la propuesta de Roca, Márquez y Sanmartí. De manera similar, Romero y Pulido (2015) en su investigación sobre el fortalecimiento de las habilidades científicas: observar y preguntar en estudiantes de cuarto grado, ejecutan una adaptación de las categorías de observación propuestas por Santelices. A nivel de la argumentación, Tamayo (2012), en su estudio sobre la argumentación como elemento clave del pensamiento crítico para el estudiantado de cuarto y quinto grado, establece niveles de argumentación a partir de las matrices argumentativas de Toulmin.

\section{Transformaciones en el diseño curricular de Ciencias Naturales}

Es clara la orientación en materia curricular establecida por el Ministerio de Educación Nacional, sin embargo, su implementación empieza a ser modificada desde el diseño curricular cuando las instituciones educativas deben elaborar sus propios marcos curriculares. Todas las instituciones no ponen en marcha los mismos contenidos y procesos debido a la multiplicidad de factores que influyen para planear e implementar sus distintas apuestas curriculares, pues se empieza a configurar cierta singularidad a lo que se enseña. Es de tener en cuenta que el currículo se construye desde el saber pedagógico, el cual implica la intervención de distintas fuentes de saber y conocimiento como son la epistemológica, la psicopedagógica, la sociocultural, la filosófica y la pedagógica (Rivera, 2010). Para tal fin, es indispensable examinar el marco legal que faculta a las instituciones educativas para ajustar el currículo de Ciencias y, por consiguiente, las posibles modificaciones que se van generando en sus componentes.

\subsection{La flexibilidad del currículo desde el marco legal}

En primer lugar, el Estado mediante la Ley General de Educación o Ley 115 (1994), en sus artículos 23 y 31, determinó las Ciencias Naturales y Educación Ambiental como un área fundamental y obligatoria del conocimiento que se cursa durante los nueve años de educación básica y los dos años de educación media. Posteriormente, el Decreto 1860 (1994), en su capítulo V, fijó las orientaciones curriculares para la construcción del currículo, la organización de las áreas, el desarrollo de las asignaturas, la adopción del currículo, y dio autonomía a las instituciones educativas para establecer las intensidades horarias. Como complemento, el Decreto 1850 (2002), en su artículo $2^{\circ}$, definió para establecimientos públicos oficiales el tiempo para cumplir con el Proyecto Educativo Institucional (PEI) y el plan de estudios durante un periodo de 40 semanas lectivas con una intensidad horaria anual para básica primaria de 1000 horas y 1200 horas para básica secundaria y media, hecho que, implica dedicar para desarrollar las áreas obligatorias como mínimo un $80 \%$ de dichas intensidades; en el caso de los establecimientos educativos privados, en la Resolución 1730 (2004) se establecieron las mismas intensidades horarias anuales.

Ahora bien, la normatividad vigente alusiva a la flexibilidad curricular es amplia, por lo que, se enuncian las principales normas relacionadas con el diseño curricular en Ciencias. Un primer aspecto es lo definido por el Decreto 1962 (1969), en sus artículos $6^{\circ}$ y $7^{\circ}$, respecto a la enseñanza media diversificada en el país, pues faculta a los Institutos de Educación Media Diversificada para organizar sus programas según áreas de énfasis como la académica, industrial, agropecuaria, comercial y, técnico social; cada una de estas áreas, a su vez, se 
subdivide en modalidades. En uno de los parágrafos, el decreto manifiesta que el gobierno reglamentará la intensidad horaria, proceso que debe hacerse según la modalidad de enseñanza.

Otra situación que implica hacer ajustes curriculares es la metodología de Escuela Nueva para áreas rurales, específicamente, el Decreto 1490 (1990), en su artículo 2º expresa que las instituciones prioritariamente de educación básica que la implementen podrán desarrollar guías y adecuar el currículo a las características de cada región, necesidades e intereses del estudiantado y progenitores o responsables de familia de conformidad con los criterios básicos que para su aplicación establece el Ministerio de Educación Nacional. En consecuencia, los contenidos, niveles de profundización y procesos metodológicos para las clases de Ciencias Naturales deben ajustarse según el contexto.

Luego con el Decreto 804 (1995), en sus capítulos I y III, se establecen las pautas para la atención educativa de grupos étnicos y determina los principios de la etnoeducación, entre los cuales, merece ser destacada la flexibilidad de los procesos etnoeducativos acordes con los valores culturales, necesidades y particularidades de estos. Además, define orientaciones curriculares fundamentadas en la territorialidad, la autonomía, la lengua, la concepción de vida de cada pueblo, su historia e identidad según sus usos y costumbres.

Un aspecto nuevo y diferenciador en el proceso curricular corresponde a los programas de alfabetización, educación formal y media de personas adultas, para ello, el Decreto 3011 (1997), en su capítulo III, manifiesta que las instituciones educativas con esta población deben atender las orientaciones curriculares generales que expidan las entidades territoriales certificadas en educación y el Ministerio de Educación Nacional, la duración de estos programas tendrá la flexibilidad necesaria según las características regionales y los grupos humanos por atender. El alcance de los propósitos de aprendizaje para este grupo poblacional difiere dado que cada ciclo lectivo tiene una duración no menor a 800 horas anuales y, además, se efectúa de forma presencial, semipresencial, abierta y a distancia, así que, influye en la cantidad de horas anuales de estudio formal.

En lo que concierne a los Colegios Internacionales, el Decreto 2832 (2005), en su artículo $6^{\circ}$, expresa que estas instituciones educativas pueden establecer en su Proyecto Educativo Institucional, la estructura u organización de los planes de estudio y la definición de los criterios para la evaluación del rendimiento del educando que rigen en el Estado con el cual se haya celebrado el convenio o acuerdo.

Otra situación particular hace alusión a la necesidad de los establecimientos educativos de organizar, flexibilizar y adaptar el currículo, el plan de estudios y los procesos de evaluación para la población en situación de discapacidad. La población educativa a la que aplica esta normatividad corresponde a estudiantes con discapacidad cognitiva, motora y autismo; alumnado sordo usuario de Lengua de Señas Colombiana (LSC); sordos usuarios de lengua castellana; ciegos, con baja visión y sordociegos; y capacidades con talentos excepcionales (Decreto 366, 2009). En ese sentido, en el año 2017 se plantearon los lineamientos respecto a los Planes Individuales de Ajustes Razonables (PIAR) que se deben implementar con este tipo de población (Decreto 1421, 2017).

Por otro lado, la Ley 1388 (2010), en su artículo $2^{\circ}$, contempla el apoyo académico especial para la población menor de 18 años matriculada en establecimientos educativos para los niveles de preescolar, básica y media, y que se encuentre hospitalizada en el sector público o privado, entre los cuales, se mencionan los enfermos de cáncer, entre otros. Para esto, las prácticas pedagógicas y didácticas deben adaptarse a los ritmos propios de aprendizaje y las condiciones particulares e individuales de la población menor de 18 años a la que se hace referencia (Decreto 1470, 2013).

\subsection{Ajustes en los componentes del curriculo de Ciencias Naturales}

Autores como Lawrence Stenhouse, Stephen Kemmis, Gimeno Sacristán, Raewyn Connell, entre otros, hacen planteamientos sobre el currículo, los cuales, sirven para comprender cómo se puede reformular su concepción desde la prescripción hasta su realidad (Barraza, 2018). Para Posner (2005), el currículo es concebido: 
Como alcance y secuencia de las prácticas educativas, como programa o programación de estudio, como esquema de contenidos, como agrupación de estándares, como libro de texto y como ruta general de estudio, hasta consideraciones generales que lo asimilan a un conjunto de experiencias planeadas. (p. 6)

En principio, no existe una receta para diseñarlo, hay modelos que deben guiarse desde un sentido amplio de todos los participantes del proceso sin enfocarse solo en los componentes individuales.

En algún momento del año escolar, generalmente al principio, las instituciones educativas generan espacios entre docentes para la revisión, ajuste y actualización de los componentes curriculares, el proceso parte de lo construido en materia curricular. Para ello, cada institución define los criterios para el análisis a partir de las reuniones entre docentes que se hacen por áreas de estudio, asignaturas, grados, niveles o situaciones especiales. Sin embargo, el cumplimiento de este propósito tiende a desarrollarse de forma parcial y depende de cada institución, algunas dificultades en el proceso corresponden a la limitación del tiempo establecido para ejecutarlo, las concepciones del currículo por parte del personal docente de ciencias, la concepción de ciencia y de lo que se debe enseñar, entre otras causas. Dadas las características de las Ciencias Naturales, la revisión del currículo desde grados inferiores hasta superiores y su articulación con los otros campos del conocimiento, demanda tiempo considerable; en muchos casos, no se alcanza a revisar todo o se hace sin el suficiente análisis. Puede presentarse el caso de que se haga de forma fraccionada en virtud de la operatividad para hacerlo, es decir, se tenga un panorama solo por niveles: preescolar, básica primaria, básica secundaria y media vocacional, no un panorama completo. Hecho que se relaciona con la revisión inadecuada de la articulación del currículo de Ciencias con el de otros campos de estudio. $\mathrm{Al}$ respecto, es indispensable hacer una revisión de lo que sucede con los componentes requeridos para el diseño curricular, visto desde los factores que inciden en la transformación del currículo de Ciencias.

\subsubsection{Propósitos de aprendizaje en Ciencias Naturales}

Las instituciones educativas parten del macrocurrículo con el marco curricular internacional y nacional para definir los propósitos de aprendizaje, no obstante, dichos propósitos en Ciencias son modificados a partir del contexto en el mesocurrículo y, pueden alterarse según el perfil y experiencia del cuerpo docente.

Los propósitos de aprendizaje en Ciencias Naturales no son exactamente los mismos en un contexto urbano o rural, esto es, tienen relación con el énfasis de las instituciones educativas; es de esperar, en colegios rurales un énfasis a nivel agropecuario o técnico y con alto contenido de temáticas asociadas a la educación ambiental. En cambio, en el sector urbano existe una amplia gama de contextos que se relacionan con el nivel socioeconómico y por supuesto, con el énfasis de las instituciones, a saber: técnico, académico, industrial, comercial. En tal sentido, la normatividad nacional permite a las instituciones educativas ajustar los planes de estudio y las intensidades horarias anuales de acuerdo con su contexto y énfasis, por consiguiente, los propósitos empiezan a diferenciarse entre instituciones en términos de tiempo o profundización.

Según el tipo de población atendida, para el caso del estudiantado en situación de discapacidad, los objetivos se proyectan tanto para los contenidos como para las competencias que se pretende desarrollar y fortalecer, esto requiere hacer ajustes en las competencias de ciencias en relación con el grado o nivel del alumnado. Este proceso es adelantado generalmente por educadores especiales en consenso con docentes titulares de las clases; no obstante, hay inconvenientes en el diseño de estos propósitos por parte de titulares a partir de la aceptación en sus aulas de estudiantes en esta situación, por tal razón, no siempre se contempla la definición de propósitos y valoraciones diferenciados desde una misma actividad, hecho que aplica también para aulas multigrado. Para Arenas y Sandoval (2013), "la flexibilización curricular implica mantener los objetivos generales propuestos para todos los escolares en el currículo, sin embargo, en las prácticas de aula, la flexibilización conlleva ajustar los procesos de acuerdo a las características de aprendizaje de los escolares" (p. $150)$. 
Igualmente, la forma como el profesorado de Ciencias conciben el currículo influye en el proceso, en muchas instituciones hay docentes por varios años que tienen la misma asignación académica y en los mismos grados, por lo tanto, tienden a considerar que lo planteado en el plan de estudios está bien, en consecuencia, los propósitos de aprendizaje y los contenidos no cambian por muchos años. Es decir, resultan concibiendo el currículo como estático o permanente.

Por último, la concepción de las ciencias y su enseñanza por parte del grupo docente incide en la determinación de los propósitos, en este caso, han ingresado al sector público oficial profesionales sin una formación específica en enseñanza de las ciencias según las convocatorias de concurso abierto de méritos para proveer los empleos vacantes de docentes, por lo cual, su concepción de Ciencias o de una asignatura en particular de las ciencias es variada e influye en lo que consideran debe enseñarse. Por ejemplo, en básica secundaria y media vocacional es posible encontrar licenciados en biología con asignación académica en química o viceversa, licenciados en matemáticas como docentes de física o a la inversa, químicos ejerciendo como docentes de química, entre otros casos. En algunos establecimientos privados, esta situación se presenta con la asignación académica para licenciados debido a la organización de las instituciones de acuerdo con el tamaño de la población atendida. Igualmente, en el nivel de básica primaria, por lo general, el profesorado tiene una asignación académica que comprende la mayoría de asignaturas y, cambia o no de los grados a cargo con los años, eso está determinado por la organización de cada establecimiento educativo, es decir, son licenciados, pero no tienen una formación específica en Ciencias Naturales o Tecnología.

\subsubsection{Selección y organización de los contenidos}

La imagen de ciencia que tiene el profesorado es producto de su formación y experiencia a lo largo de los años y, tiene un efecto a la hora de enseñar pues influye en los contenidos seleccionados, las actividades desarrolladas, el método implementado, la forma de evaluar y los recursos que utilizan (Pacífico, 2017). En tal sentido, el tipo de formación disciplinar en el cuerpo docente es un factor que incide en la selección de contenidos o en el nivel de profundización proyectado para enseñar; si el currículo y las ciencias se consideran como estáticos, entonces, el plan de estudios es el mismo por muchos años, visto de otra forma, no se considera que diversos conceptos se están reformulando conforme surgen nuevos conocimientos producto de descubrimientos científicos y desarrollos tecnológicos (Quse y De Longhi, 2005). A pesar de que hay unos lineamientos curriculares para determinar la selección de los contenidos, resulta muy complejo desarrollarlos literalmente debido a la naturaleza cambiante de las ciencias y a las múltiples particularidades de las instituciones educativas.

Por otra parte, la libertad de cátedra que ejerce el profesorado da lugar a lecturas contextuales de la realidad donde los lineamientos curriculares institucionales son adaptados a cada situación. Esto siempre con el riesgo de nivelar por lo bajo a estudiantes y de no poder profundizar las problemáticas y temáticas planificadas, además, ese nivel de autonomía puede desviarse de lo que realmente se mide en las pruebas estandarizadas a nivel nacional e internacional implicando que los contenidos y habilidades científicas desarrollados no se correlacionen con los propósitos de estas pruebas.

En ese contexto, para estudiantes en situación de discapacidad no siempre se reajustan los contenidos según su tipo de condición, puesto que en muchos casos se desconocen sus reales necesidades. Hay dificultades en el diseño de los planes individuales de ajustes razonables y, por ende, en algunos casos no se proyecta un desarrollo de procesos diferenciados. Adicionalmente, cuando el alumnado está en aulas de inclusión, un buen número de docentes no tienen claro cuáles discapacidades están asociadas a procesos intelectuales u otros procedimientos, lo cual deriva en el desarrollo inadecuado de los contenidos, o bien no se contempla que estos pueden enseñarse o aprenderse en distintos tiempos. En consecuencia, es probable que los contenidos no consideren opciones de percepción, lenguaje y símbolos, y comprensión propios de esta población (Arenas y Sandoval, 2013). Al revisar un caso particular como el de estudiantes con diferencias auditivas se evidencia 
que para la enseñanza de las ciencias se requiere de un trabajo previo de articulación entre docentes e intérpretes, en tal sentido, los procesos comunicativos podrían alterarse dado que algunos términos técnicos no tienen una seña específica equivalente, o bien, se deben ajustar con las señas para expresarlos.

\subsubsection{Estrategias metodológicas}

La formación pedagógica, la visión de docentes sobre las prácticas, la infraestructura y la experiencia son aspectos que condicionan las estrategias metodológicas proyectadas, la realidad muestra que estos factores influyen al momento de planear las clases.

Como ya se dijo, hay docentes de ciencias que tienen una formación profesional pura sin fundamentos en pedagogía, los adquieren a través de varios años de ejercicio, esto significa que se distorsiona parte del proceso educativo como consecuencia del perfil docente, pues la calidad de la enseñanza de las ciencias es afectada y, por lo tanto, el nivel de aprendizaje del estudiantado.

Hay estrategias metodológicas orientadas hacia las prácticas, en cuyo caso, se presentan diferencias de aprendizaje relacionadas con los objetivos fijados por cada docente para estas, no se aprende lo mismo de un laboratorio tradicional tipo receta con resultados predeterminados que de un laboratorio con procedimientos generados por el alumnado y resultados indeterminados o diferentes posibilidades de solución. El trabajo práctico en la enseñanza de las ciencias depende de la visión que tiene el/ la docente de lo que es la práctica e influye en la definición de los objetivos de un laboratorio, pues deben corresponder con los objetivos de la enseñanza de las ciencias y tener conexión con los estilos de enseñanza del laboratorio, autores como Domin, Moreira y Levandowski, y Kirschner plantean estilos de enseñanza para los laboratorios de ciencias (Flores, Caballero y Moreira, 2009).

Un aspecto relacionado con el carácter teórico o no de las clases es la infraestructura y recursos disponibles, hay docentes bastante teóricos por razones asociadas a la falta de laboratorio en sus instituciones educativas u otras causas, docentes con un mayor enfoque para desarrollar prácticas que implican el uso de laboratorios o de los recursos con los que cuenta cada institución, o bien, aquellos que desarrollan procesos teóricos y prácticos ajustados según la temática y propósito de aprendizaje.

En el caso de estudiantes en situación de discapacidad se deben contemplar los estilos y ritmos de aprendizaje, los tiempos para lograr desarrollar las competencias esperadas, y el diseño de actividades que promuevan la motivación y el reconocimiento de individualidades (Arenas y Sandoval, 2013). Sin embargo, no siempre se consideran esos aspectos por la falta de información oportuna entre docentes y educadores/as especiales y, las dificultades mencionadas anteriormente.

\subsubsection{Sistema de evaluación}

El cuerpo docente con escasa o sin formación pedagógica tiene un conocimiento general sobre tipos, técnicas e instrumentos de evaluación, es posible que articule parcialmente procesos cognitivos, procedimentales y actitudinales desde lo planeado hasta lo evaluado, visibilizados en el diseño de los instrumentos de evaluación que utilizan. Según Santos (2003), uno de los componentes básicos de la evaluación es la comprobación de los aprendizajes, por lo cual, puede llegar a convertirse en un factor diferenciador si no se hace de forma objetiva. En otras palabras, si la persona docente al evaluar un propósito de aprendizaje expresa que fue aprobado y en la realidad, el estudiantado presenta vacíos conceptuales, entonces, se empieza a formar una ruptura entre el currículo prescrito y el aprendido. En relación con los procesos de evaluación para estudiantes en situación de discapacidad, muchos/as docentes no conocen procesos apropiados para evaluarlos, por lo que resultan desconociendo sus diferencias y ritmos de aprendizaje, no tienen en cuenta los niveles de aprendizaje que podrían alcanzar. 


\section{TRANSFORMACIONES EN LA IMPLEMENTACIÓN DEL CURRÍCULO}

La implementación del currículo implica cambios en el proceso producto de situaciones asociadas fundamentalmente al contexto, la forma en que docentes de ciencias implementan su conocimiento profesional y las dinámicas propias de cada institución educativa. Todo esto repercute en el cumplimiento de los propósitos de aprendizaje durante el año lectivo, ya que se relaciona con el tiempo realmente efectivo de las clases y, por supuesto, con los procesos de aprendizaje pues se van generando diferencias entre la comunidad estudiantil.

El contexto implica hacer modificaciones desde diferentes niveles organizacionales que atraviesan lo administrativo, operativo y los procesos de aula. Los ajustes se hacen en función de las condiciones específicas de la escuela considerando las características sociales, culturales y económicas del sector donde se localiza, y las características particulares de la población de estudiantes. La diversidad de contextos se refleja en la visualización de contenidos y los distintos niveles de profundización, puesto que cambian aspectos específicos como la intensidad horaria, el plan de estudios según el énfasis de las instituciones, la disponibilidad de recursos, entre otros. Para citar algunos casos, entre instituciones públicas o privadas hay diferencias en virtud de la existencia de espacios específicos para la enseñanza de las ciencias como laboratorios, viveros, rincones de ciencias, aulas de informática, aulas especializadas, etc. La disponibilidad de estos espacios repercute en la calidad de los procesos desarrollados por docentes y estudiantes, no es lo mismo contar con un laboratorio para biología, física o química bien dotado y un aula de informática con suficientes equipos y actualizados en las grandes ciudades que, carecer de un espacio para desarrollar las prácticas experimentales en zonas rurales o disponer de un laboratorio subutilizado. También hay diferencias entre el sector privado, algunos establecimientos educativos tienen suficientes recursos para desarrollar estas prácticas, mientras que otros, prácticamente solo cuentan con espacios para las clases regulares. De igual manera, al observar el contexto de aula se hallan cambios producto de circunstancias que influyen en el curso de las clases, "un mismo profesor enseña de forma diferente, un mismo contenido, a diferentes alumnos dependiendo de las condiciones particulares de los estudiantes y de la escuela" (Barnett y Hodson, citados por Valbuena, 2007, p.70).

En el caso de las instituciones educativas con estudiantes en situación de discapacidad, algunas dificultades para la puesta en marcha del proceso se presentan porque no siempre los colegios disponen del personal profesional en el momento oportuno, o bien, la resistencia presentada por algunos/as docentes del sector público oficial para flexibilizar sus procesos ya sea porque no se sienten en capacidad de atender a la población en dicha situación o porque consideran que aplicar los Planes de Ajustes Razonables les implica mucho tiempo de preparación y atención en comparación con lo que se hace con estudiantes sin tal situación. Del mismo modo, en lo referente al proceso desarrollado con estudiantes menores de 18 años hospitalizados se encuentran dificultades asociadas con la asignación oportuna de personal docente en las entidades de salud para hacer el acompañamiento efectivo a estudiantes, este personal no se asigna para cada campo del conocimiento sino que un profesional atiende todas las disciplinas del conocimiento; también, se presentan problemas de comunicación eficiente entre instituciones educativas y de salud para articular propósitos de aprendizaje y conocer la valoración oportuna de los procesos desarrollados por el estudiantado.

En cuanto a la implementación del conocimiento profesional del cuerpo docente, Valbuena (2007), hizo una revisión desde diferentes autores sobre los componentes de este conocimiento: pedagógico, contextual, del contenido disciplinar y el didáctico de contenido. Algunos cambios en la materialización del currículo de las ciencias están asociados a unos o todos los componentes del conocimiento profesional de cada docente, se relacionan con sus perfiles profesionales y cómo se han expresado son posiblemente producto de la forma de concebir el currículo y las ciencias, hecho que incide en el modo de implementar cada uno de los componentes del currículo. A nivel conceptual, los contenidos disciplinares que se enseñan en ciencias podrían cambiar entre docentes con formaciones académicas diferentes, un caso particular es la enseñanza de las ciencias en niñas y niños pequeños porque se podrían presentar experiencias descontextualizadas, sin sentido y con 
dificultades, y encontrarse docentes con errores conceptuales de base y falta de dominio de contenidos (Daza, Orellana y Quintanilla, 2011). También, existen cambios asociados a los estilos de enseñanza del cuerpo docente de ciencias que influyen en el desarrollo de los procesos curriculares y se reflejan en el aprendizaje de las ciencias, un/a docente tradicionalista o tipo cátedra con métodos centrados en el mismo profesorado, con métodos orientados hacia el estudiantado o con un alto grado de énfasis en desarrollar sus clases ya sea desde la práctica, las fórmulas, los modelos, las lecturas u otras maneras, influye en el modo en que el alumnado comprende las ciencias.

Adicionalmente, es de tener en cuenta que el personal docente no es un simple actor que implementa lo planificado en materia curricular, en relación con esto, Pérez (2015) plantea:

La condición esencial del docente como intelectual de la educación, quien al asumir críticamente su labor educativa se configura como protagonista determinante en la cualificación formativa de los aprendices y como gestor colaborativo de los desarrollos curriculares en los diferentes niveles. (p. 78)

Desde ese punto de vista, el profesorado de ciencias va más allá de ejecutar el currículo, pues incentiva y orienta la práctica pedagógica independientemente de su rol, no solamente se limita a implementar lo definido en materia curricular, sino que dentro de los procesos que desarrolla con su práctica deja su sello personal al influir en la percepción de las ciencias que tenga el estudiantado, en los contenidos que están asimilando y, en su formación integral (Rozo, 2018). Para Chona (2009), una reflexión sobre el ejercicio pedagógico del personal docente de ciencias expresa:

Una cosa es enseñar con modelos racionales, llamémoslos con intención científica, y otra cosa es enseñarles ciencia a los niños a la manera de memorización de datos e información sin significado para ellos. Quien quiera enseñarle ciencia a un niño así, creo que se está equivocando; el que debe ser un científico es el maestro de esos niños, dado que debe ser un conocedor profundo e investigador de la condición del niño, de la pedagogía, de los saberes que para esos niveles son posibles de aprender o construir. (p. 2)

Por último, las dinámicas propias de cada institución educativa contribuyen directa o indirectamente en el cumplimiento de los procesos curriculares causando cambios en los tiempos programados para desarrollar las actividades y los contenidos y, la aplicación de las evaluaciones. Algunas de estas situaciones corresponden al ausentismo docente, el cumplimiento de actividades institucionales programadas según el calendario escolar, el desarrollo de actividades no programadas o extracurriculares las cuales surgen en virtud de alianzas con entidades externas que apoyan diferentes procesos escolares, los recesos del magisterio en el sector público oficial, el conflicto en determinadas regiones del país, problemas particulares asociados a las plantas físicas y los servicios públicos, el ausentismo continuo o no del estudiantado, el tiempo dedicado por cada docente para organizar y controlar el comportamiento de estudiantes durante sus clases que varía entre sesiones para un mismo grupo o entre diferentes grupos, el desarrollo de las temáticas durante las clases cuando se generan y resuelven las dudas o se profundiza según el interés presentado por el alumnado, hecho que, inclusive puede resultar desviándose de su propósito inicial y, el uso del internet a través de las redes sociales u otros medios de información podría causar una conceptualización distorsionada en Ciencias, debido a que la mayoría de estudiantes no ha tenido una orientación adecuada para el acceso a fuentes confiables de información, entre otros aspectos.

\section{CONSIDERACIONES FINALES Y PROPUESTAS}

Una de las características del currículo debe ser su flexibilidad según la diversidad de situaciones que se presentan en las instituciones educativas. Muchos elementos son cruciales porque le confieren su naturaleza cambiante, entre los cuales, se destacan factores exógenos como el contexto social, cultural, económico y, factores endógenos como el contexto institucional, de aula y la forma como docentes lo conciben, diseñan e implementan. 
A continuación, se señalan algunos elementos por considerar para la implementación del currículo de Ciencias Naturales:

- Hay transformaciones del currículo asociadas al conocimiento profesional del cuerpo docente de ciencias, las cuales, deben ser objeto de estudios detallados para docentes en primaria y secundaria con el fin de conocer las transformaciones derivadas del perfil profesional, esto es, tanto para quienes tengan una asignación académica en su respectiva asignatura como para quienes tienen asignación en lo que no han sido formados.

- El contexto es fundamental pues influye significativamente en la transformación de los procesos curriculares. Para su formulación resultaría útil verificar su real alcance, los diferentes tipos de contexto, las matrices o niveles de desarrollo para las competencias científicas propuestas por diversos autores, las taxonomías para la formulación de objetivos, el tipo de población atendida, entre otros. Igualmente, al examinar el contexto específico o de aula es necesario hacer ajustes a nivel del microcurrículo, autores como Bermúdez y Longhi (2012) plantean que los contextos situacional, lingüístico y mental están presentes en las clases; por lo tanto, deben ser contemplados para definir los propósitos.

- La malla curricular es un marco de referencia para evaluar el currículo, su construcción debe ser producto de un trabajo colectivo de docentes que parte tanto de los contenidos como de las competencias. Pues bien, los ejes curriculares pueden plantearse desde diferentes miradas, por ejemplo, a partir de ejes independientes y transversales relacionados con las habilidades de pensamiento científico.

- Desarrollar los procesos de pensamiento o competencias científicas debe estar alineado desde el diseño curricular hasta su materialización; para ello, es fundamental incorporar elementos de dichos procesos en los propósitos de aprendizaje, las estrategias metodológicas y, los procesos de evaluación. Igualmente, el desarrollo por competencias debe comprender los niveles o categorías propias para el desarrollo de los procesos de pensamiento que se pretenden desarrollar o fortalecer en el estudiantado. Y desarrollarse de forma progresiva en un mismo grado o entre todos los grados educativos.

- Muchas estrategias y actividades complementarias que se desarrollan desde el campo de las Ciencias Naturales en las instituciones educativas no están articuladas con el plan de estudios, en algunos casos, se efectúan de forma aislada. Por consiguiente, es indispensable articular curricularmente estrategias como el Proyecto Ambiental Escolar (PRAE), ferias de ciencias, salidas pedagógicas a museos, parques ecológicos o sitios de interés ambiental y alianzas con entidades que apoyan procesos educativos de las ciencias o la educación ambiental, pues sirven para retroalimentar las prácticas de aula y brindar orientaciones para el fortalecimiento del currículo de ciencias al promover el desarrollo de sus competencias.

- La evaluación es un proceso fundamental que influye en la consolidación del currículo prescrito. Una estrategia útil para la evaluación de los aprendizajes es el diseño y uso de rúbricas de evaluación como instrumento que aporta en la aplicación de la evaluación formativa pues contempla distintos niveles de aprendizaje para las competencias científicas y los conceptos. Esta estrategia ayuda a mejorar los procesos de evaluación para estudiantes en o sin situación de discapacidad.

- El currículo de ciencias se revisa periódicamente, sin embargo, el proceso se hace de forma parcial por lo que sus ajustes tienden a ser incompletos. En consecuencia, evaluar periódicamente el currículo de Ciencias Naturales según la definición de claros criterios para su valoración e indicadores del proceso desarrollado, que implique hacer actualizaciones a nivel del mesocurrículo y el microcurrículo. Esto debe hacerse con base en los avances que la ciencia y la tecnología tienen, las competencias científicas y, demás aspectos inherentes a la evaluación.

\section{REFERENCIAS}

Arenas, F. y Sandoval, M. (2013). Procesos de flexibilización y diversificación curricular: Nuevo retos del sistema educativo colombiano para favorecer los procesos de participación en contextos escolares de personas con 
discapacidad. Horizontes Pedagógicos, 15(1), 147 - 157. Recuperado de https://horizontespedagogicos.ibero.ed u.co/article/view/421

Barraza, N. (2018). El Currículum, Análisis y Reformulación del Concepto. Dictamen Libre, 1(22), 113-118. doi: h ttps://doi.org/10.18041/2619-4244/dl.22.5032

Bermúdez, G. y De Longhi, A. (2012). Capítulo I. El conocimiento didáctico de contenidos biológicos de Ecología. En R. Calixto. (Coord). Experiencias Latinoamericanas en educación ambiental (pp.17-35). Monterrey, México: Colegio de Estudios Científicos y Tecnológicos del Estado de Nuevo León (CECyTE NL).

Casanova, M. (2006). Diseño curricular e innovación educativa (2da ed.). Madrid, España: Editorial La Muralla, S.A.

Chona, G. (2009). Acerca de la enseñanza de las ciencias. Una visión personal. Revista Biografía, 2(1). 124-128. doi: https://doi.org/10.17227/20271034.vol.2num.2bio-grafia124.128

Daza, S., Orellana, M. y Quintanilla, M. (2011). La ciencia en las primeras edades como promotora de competencias de pensamiento científico. En S. Daza y M. Quintanilla. (Eds). La enseñanza de las ciencias naturales en las primeras edades (pp 59-82). Barrancabermeja, Colombia: GRECY INYUBA.

Decreto 1962. Diario Oficial No. 32953. Bogotá, Colombia, 10 de diciembre de 1969. Recuperado de https://bit.ly /2U10xEX

Decreto 1490. Diario Oficial No. 39461. Bogotá, Colombia, 9 de julio de 1990. Recuperado de https://bit.ly/2B3v dOV

Decreto 1860. Diario Oficial No. 41473. Bogotá, Colombia, 3 de agosto de 1994. Recuperado de https://bit.ly/2X7 $\mathrm{m} 7 \mathrm{ct}$

Decreto 804. Diario Oficial No. 41853. Bogotá, Colombia, 18 de mayo de 1995. Recuperado de https://bit.ly/2TM YFPI

Decreto 3011. Diario Oficial No. 43202. Bogotá, Colombia, 19 de diciembre de 1997. Recuperado de https://bit.ly /2ZFA8jv

Decreto 1850. Diario Oficial No. 44901. Bogotá, Colombia, 15 de agosto de 2002. Recuperado de https://bit.ly/3e $\mathrm{rEcrO}$

Decreto 2832. Diario Oficial No. 46003. Bogotá, Colombia, 16 de agosto de 2005. Recuperado de https://bit.ly/2A behWq

Decreto 366. Diario Oficial No. 47258. Bogotá, Colombia, 9 de febrero de 2009. Recuperado de https://bit.ly/2M5 $5 \mathrm{GHw}$

Decreto 1470. Diario Oficial No. 48849. Bogotá, Colombia, 12 de julio de 2013. Recuperado de https://bit.ly/36y QonS

Decreto 1421. Diario Oficial No. 50340. Bogotá, Colombia, 29 de agosto de 2017. Recuperado de https://bit.ly/2Z IczXf

Flores, J., Caballero, M. y Moreira, M. (2009). El laboratorio en la enseñanza de las ciencias: Una visión integral en este complejo ambiente de aprendizaje. Revista de investigación. 33(68), 75-111. Recuperado de https://dialnet .unirioja.es/servlet/articulo?codigo $=3221708$

García, S. y Furman, M. (2014). Categorización de preguntas formuladas antes y después de la enseñanza por indagación. Praxis \& Saber. 5(10), 75-91. doi: https://doi.org/10.19053/22160159.3023

Glatthorn, A., Boschee, F., Boschee, B. y Whitehead, B. (2018). Chapter I. The Nature of Curriculum. Curriculum Leadership: Strategies for Development and implementation ( $5^{\text {th }}$ ed.). USA: Sage Publications, Inc.

Ley 115. Diario Oficial No. 41214. Bogotá, Colombia, 8 de febrero de 1994. Recuperado de https://bit.ly/3d8xve1

Ley 1388. Diario Oficial No. 14721. Bogotá, Colombia, 26 de mayo de 2010. Recuperado de https://bit.ly/2M7c29n

Ministerio de Educación Nacional, Colombia [MEN]. (1998). Lineamientos curriculares en Ciencias Naturales y Educación Ambiental. Bogotá: Ministerio de Educación Nacional.

Ministerio de Educación Nacional, Colombia [MEN]. (2004). Estándares Básicos de Competencias en Ciencias Naturales y Ciencias Sociales. Bogotá: Ministerio de Educación Nacional. 
Ministerio de Educación Nacional, Colombia [MEN]. (2016). Propuesta de estructura y fundamentación de los DBA. Componente Ciencias Naturales. Bogotá: Ministerio de Educación Nacional.

Ministerio de Educación Nacional, Colombia [MEN]. (2017). Mallas de aprendizaje. Documento para la implementación de los DBA. Bogotá: Ministerio de Educación Nacional.

Pacífico, A. (2017). La imagen de las ciencias en el diseño curricular y su incidencia en la formación de los profesores de Biología. Conferencia llevada a cabo en $X$ Congreso Internacional sobre investigación en didáctica de las ciencias. Sevilla, España. Recuperado de https://bit.ly/3c3B97u

Pérez, L. (2015). La práctica docente como experiencia microcurricular sistémica. Revista Internacional de Pedagogía y Curriculo, 2(2), 75 - 86. Recuperado de https://bit.ly/2IMS4ik

Posner, G. (2005). Análisis del currículo. (3a ed.). México, D.F.: Mc Graw Hill Interamericana.

Quse, L. y De Longhi, A. (2005). ¿Qué dicen los docentes de Biología del nivel medio sobre la educación CTS? Diagnóstico en Córdoba, Argentina. Revista Electrónica de Enseñanza de las Ciencias, 4(2), 1 - 14. Recuperado de http://reec.uvigo.es/volumenes/volumen4/ART7_Vol4_N2.pdf

Resolución 1730. Diario Oficial No. 45593. Bogotá, Colombia, 18 de junio de 2004. Recuperado de https://bit.ly/2 $\mathrm{XCmKtD}$

Rivera, J. (2010). Cómo diseñar Planes de Estudio de las áreas fundamentales y obligatorias para la Educación Preescolar, Básica y Media. Bogotá: Editorial Magisterio.

Romero, Y. y Pulido, G. (2015). Incidencia de las rutinas de pensamiento en el fortalecimiento de habilidades cientificas: observar y preguntar en los estudiantes de grado cuarto, ciclo II del Colegio Rural José Celestino Mutis IED. (Tesis de Maestría). Universidad de La Sabana, Cundinamarca. Colombia.

Rozo, H. (2018). Modelos pedagógicos: generando transformación e innovación desde las prácticas docentes. En I. Jiménez. (Ed).Informática Educativa: Origen, naturaleza yperspectivas de su investigación (pp 85-93). Colombia: ECOE Ediciones Limitada.

Santos, M. (2003). Dime cómo evalúas y te diré qué tipo de profesional y de persona eres. Enfoques educacionales, 5(1). 69-80. Recuperado de https://bit.ly/2B6bfTL

Tamayo, O. (2012). La argumentación como constituyente del pensamiento crítico en niños. Hallazgos, 9(17), 211233. Recuperado de https://revistas.usantotomas.edu.co/index.php/hallazgos/article/view/738

Valbuena, E. (2007). El conocimiento didáctico del contenido biológico: Estudio de las concepciones disciplinares $y$ didácticas de futuros docentes de la Universidad Pedagógica Nacional (Colombia). (Tesis de doctorado). Universidad Complutense de Madrid, España.

\section{BY-NC-ND}

